\title{
Searching while loaded: Visual working memory does not interfere with hybrid search efficiency but hybrid search uses working memory capacity
}

\author{
Trafton Drew $^{1}$ • Sage E. P. Boettcher ${ }^{2}$ Jeremy M. Wolfe J $^{3,4}$
}

Published online: 9 June 2015

(C) Psychonomic Society, Inc. 2015

\begin{abstract}
In "hybrid search" tasks, such as finding items on a grocery list, one must search the scene for targets while also searching the list in memory. How is the representation of a visual item compared with the representations of items in the memory set? Predominant theories would propose a role for visual working memory (VWM) either as the site of the comparison or as a conduit between visual and memory systems. In seven experiments, we loaded VWM in different ways and found little or no effect on hybrid search performance. However, the presence of a hybrid search task did reduce the measured capacity of VWM by a constant amount regardless of the size of the memory or visual sets. These data are broadly consistent with an account in which VWM must dedicate a fixed amount of its capacity to passing visual representations to long-term memory for comparison to the items in the memory set. The data cast doubt on models in which the search template resides in VWM or where memory set item representations are moved from LTM through VWM to earlier areas for comparison to visual items.
\end{abstract}

Keywords Visual search $\cdot$ Working memory $\cdot$ Dual-task performance

Imagine that you are in a grocery store, searching for the items on your memorized shopping list. With luck, the list resides

Trafton Drew

trafton.drew@psych.utah.edu

1 University of Utah, Salt Lake City, UT, USA

2 Goethe Universitat Frankfurt, Frankfurt, Germany

3 Brigham \& Women's Hospital, Boston, MA, USA

4 Harvard Medical School, Boston, MA, USA fairly stable in your long-term memory. Your shopping task is a "hybrid search", combining visual and memory search (Schneider \& Shiffrin, 1977; Wolfe, 2012). In the midst of your search, you meet someone and exchange phone numbers but you don't have a pen, so you must hold the number in Working Memory while continuing your search. Does that Working Memory load interfere with your ongoing hybrid search and if so, what aspect of the search is perturbed? We know that working memory does interact with visual search. Two influential papers demonstrated strong evidence that under some circumstances a VWM load increases the slope of the reaction time (RT) $\mathrm{x}$ set size function, indicating that a VWM load reduces search efficiency (Oh \& Kim, 2004; Woodman \& Luck, 2004). The implication is that the act of holding information in VWM slows the rate with which we evaluate potential targets. This suggests that VWM plays a vital role in our ability to determine whether an object is a target or a distractor. More generally, it supports the idea, outlined in a number of important models of visual attention (Bundesen, 1990; Desimone \& Duncan, 1995; Logan \& Gordon, 2001; Miller \& Cohen, 2001; Wolfe, Cave, \& Franzel, 1989), that working memory plays an important part in the ability to effectively deploy visual attention. The most common notion is that the 'template' for visual search, the representation of the target, resides in VWM. This idea is supported by a growing line of research that demonstrates that attention tends to be automatically drawn to information being actively held in VWM (Downing \& Dodds, 2004; Soto, Hodsoll, Rotshtein, \& Humphreys, 2008). These results seem to predict that loading VWM would disrupt hybrid visual and memory search.

Even basic visual search requires memory to specify the current target. The target can be thought of as an attentional "template" (Olivers, Peters, Houtkamp, \& Roelfsema, 2011) or "set" (Wolfe, 1994) that must be maintained in some 
durable memory format while the search is being performed. A deluge of recent research has implicated WM as the mechanism that holds the attentional template in an activated state so that we recognize when an item matches the template. For instance, Lavie and colleagues have shown that observers are worse at ignoring distractors under WM load (Lavie, 2005). Olivers et al. demonstrated that visual search is reliably slowed if an item that is being held in working memory also appears as an irrelevant distractor in the search array (Olivers, Meijer, \& Theeuwes, 2006). Monkey neurophysiology appears to further strengthen this view. The firing rates of neurons that are sensitive to a target's features increase while that specific object is the target of a search task (Chelazzi, Duncan, Miller, \& Desimone, 1998). Finally, search efficiency decreases in the face of certain types of WM loads (Oh \& Kim, 2004; Woodman \& Luck, 2004).

Interestingly, Woodman and Luck found that WM for nonspatial information interfered with Visual Search but only when the target of search varied from trial to trial (2007). When the target template did not change, there was no effect of holding colored squares in WM on search efficiency (Woodman \& Luck, 2007). Apparently, changing the search template on each trial tapped into WM resources in a way that did not occur when the target was consistent. What would happen if, rather than changing the identity of the target template from one trial to the next, it was necessary for the observer to change the target template multiple times in a single trial? In the current set of hybrid search experiments, observers had to search for one of up to 64 possible targets. The 64-item set was held constant for a block of trials. Given the limited capacity of VWM (Luck \& Vogel, 1997; Luck \& Vogel, 2013), the full set of target templates must be held in activated long-term memory (ALTM: Cowan, 1995; Cowan, 2001), rather than working memory. Thus, if VWM is a mandatory component in visual search, then one might predict that items from the 64-member set in ALTM would be shuttled in and out of VWM during the course of a hybrid search. Following this logic, the larger the target list in ALTM, the greater would be the ALTM - VWM interaction and the greater would be the adverse effects of WM load. Forrin and Morin (1969) offer a hint to the contrary, reporting that LTM and WM do not interact in memory search. However, this work used a relatively small LTM manipulation of between 1 and 3 items and no visual search component.

In the present hybrid search experiments, involving visual and memory search, we used photorealistic objects, allowing us to employ much larger target sets, known to produce large costs in search efficiency (Wolfe, 2012). Given previous findings that VWM interferes with visual search and given the theory that the current search template resides in VWM, it comes as a surprise that, in seven experiments, we found little or no effect of loading VWM with task irrelevant material on hybrid search. However, we also found that performing a hybrid search exacted a cost on VWM performance. This cost was independent of the size of the memory set in the hybrid task.

We will use the results of these experiments to propose quite a different account of the role of VWM in visual search. We suggest that the representation of the target (or targets) of a search resides in LTM (more precisely, in ALTM). In visual search, those target representations must be compared with items in the visual stimuli. We argue that the role of WM is not to hold the templates or transfer them from LTM to earlier visual processes. Instead, we hypothesize that WM acts to pass a representation of the current object of attention from earlier visual processes to LTM where it can be compared to the target template(s).

\section{Materials and methods}

In each block of each experiment, observers were asked to memorize 2, 8, 16, or 64 photos of real-world objects (Brady, Konkle, Alvarez, \& Oliva, 2008). They then searched for the presence of a target item in visual search arrays that contained either 8 or 16 objects. On half of the trials, prior to the visual search array, observers were given a visual working memory load of three items. After responding to the search array, observers were again shown the visual working memory objects. On half of those trials, one of these items changed and the observers were asked to identify these change trials. A schematic of trials with and without a VWM load can be seen in Fig. 1. We were surprised to find no influence of a VWM load on search efficiency in Experiment 1. In the subsequent 6 experiments, we varied the nature of the VWM load in the hope and expectation of finding a situation where VWM load did interact with hybrid search. The possibility that the observers did not have enough time to encode information into the VWM in Experiment 1 led to Experiment 2, where the encoding display time was increased from 0.5 to $3 \mathrm{~s}$. Experiment 3 employed simple color square stimuli that were distinct from the visual search stimuli. Experiments 4 and 5 asked observers to perform a spatial WM task that was modeled after well-known experiments that have demonstrated a strong interaction between WM load and search efficiency (Oh \& Kim, 2004; Woodman \& Luck, 2004). Experiment 6 examined the role of the phonological loop in maintaining VWM by asking the observers to perform the task while engaged in an articulatory suppression task. Finally, Experiment 7 explored the effect of hybrid search on VWM performance, rather than the other way around, by including trials that in did not involve the hybrid search task.

Observers gave informed consent and were compensated $\$ 10$ per hour or through course credit. All observers had, at least, 20/25 acuity with correction, passed the Ishihara Color Blindness Test and were fluent speakers of English. Observers 


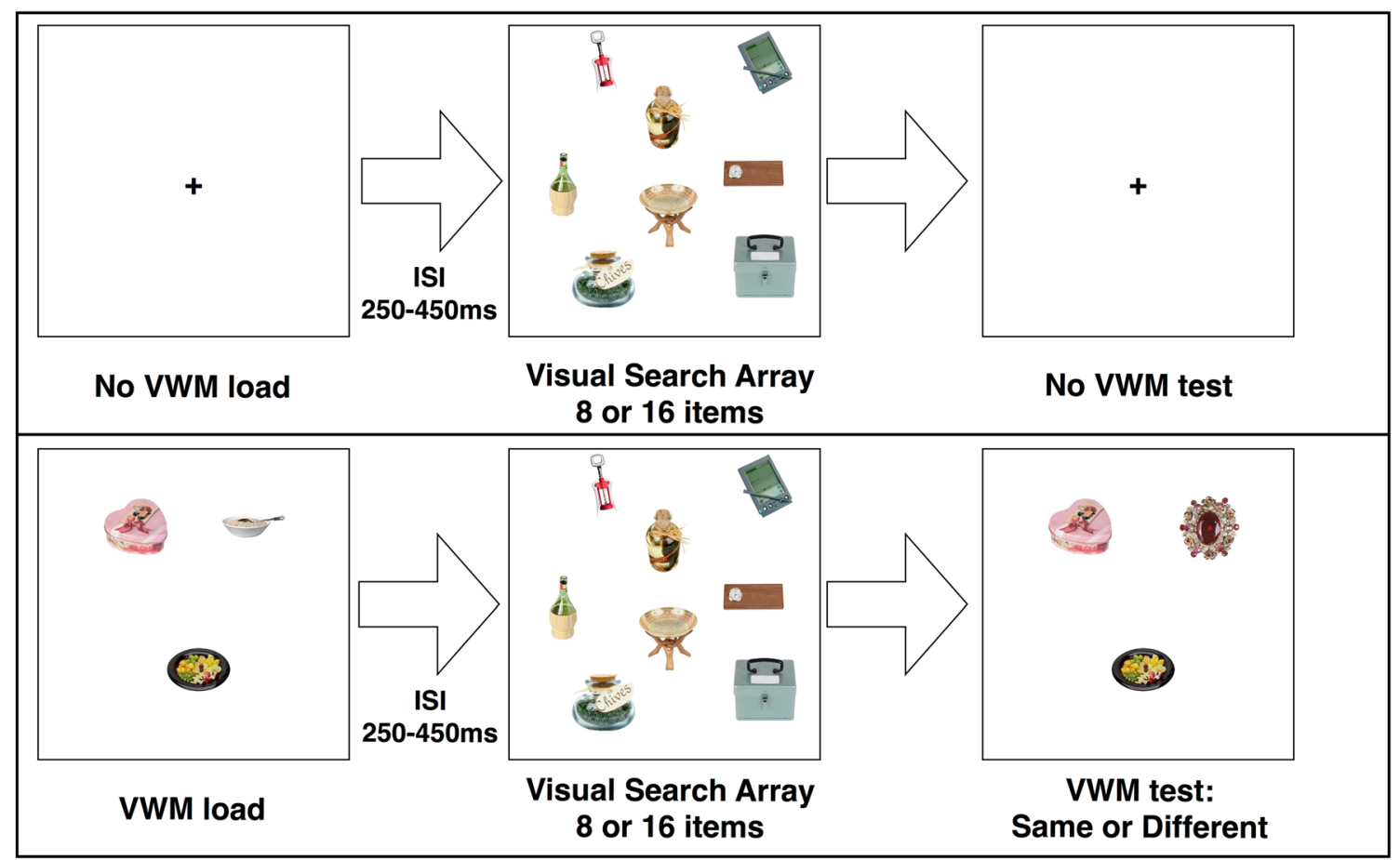

Fig. 1 Experimental paradigm schematic. Observers searched the visual search array for the presence of any of the previously memorized target set items. In Experiments 1-4 and 6, trials with and without a VWM load were interleaved. One item changed in the VWM test on $50 \%$ of the trials. The correct response in the lower example above would be "different," because one of the items changed

exceeded $30 \%$ for two other observers. One more observer was unable to finish the experiment in the allotted time.

All of the experiments began each block of trials with an identical memorization procedure similar to those described elsewhere (Drew \& Wolfe, 2014; Wolfe, 2012). Observers were asked to memorize $2,8,16$, or 64 real-world objects, which were presented individually for 3 seconds at a time. Each observer experienced all experimental conditions. Order of memory set size was randomized. All other conditions were randomized within block unless otherwise noted (as in Experiment 5). All objects were taken from a heterogeneous set of 3,000 unique photorealistic objects provided by Brady et al. (2008). During the recognition test that followed, a single object was displayed in the center of the screen and observers made "old" or "new" responses to either targets or distractor objects. Targets appeared $50 \%$ of the time. The memory test contained twice the number of trials as targets in that block. Observers were required to perform this task with at least $90 \%$ accuracy on two consecutive tests before being allowed to proceed. If performance fell below threshold, observers were retrained and retested. Block order was randomized between subjects.

After successfully learning the target set, observers completed 12 practice trials followed by 208 experimental trials. Each trial consisted of three parts. Half of the trials began with the display of the VWM load. For those trials that did not contain a VWM load, observers were shown a blank screen for the same duration as the VWM load. This was followed by 
a random blank interval between $250-450 \mathrm{~ms}$, followed by onset of the visual search array of either 8 or 16 real-world objects. Half of these arrays contained one of the target items. Nontarget "distractor" items and target items were drawn from separate sets so that an item that served as a target could never appear as a distractor. Observers indicated by key press whether a target was "present" or "absent" and were encouraged to answer as quickly and accurately as possible. After the response to the hybrid search, on trials with no VWM load, an on-screen message asked the observer to move on to the next trial. For VWM trials, the VWM test screen appeared immediately after the response to the hybrid search. The test consisted of three items, presented in the same locations as the original VWM load. For half of the trials, the identity of one of the objects changed. Throughout all of the experiments, object changes were categorical (either changing from one of seven distinct colors to another, or one unique item to another) and location changes necessitated a change from one of eight locations equally spaced around a centered circular area with a $6^{\circ}$ diameter. Observers indicated using a key press whether the test array was the "same" or "different" from the initial display. The "present" and "absent" keys were located next to each other on either the left or right side of the keyboard and the "same" and "different" keys were located together on the opposite side of the keyboard. Key assignment was counterbalanced across observers.

\section{Experiments 1 and 2: Object memory}

Thirteen observers participated in Experiment 1 and 11 participated in Experiment 2 (average age 30.7 years, 15 females). The WM displays consisted of photos of realworld objects drawn from the same superset as the targets and distractors in the hybrid search. Targets, distractors, and VWM objects were all drawn from distinct subsets of that superset. In Experiment 1, objects in the WM initial display were displayed for $0.5 \mathrm{sec}$ onds, and in Experiment 2 objects were displayed for 3 seconds.

\section{Experiment 3: Colored square memory}

Twelve observers (average age 29.3 years, 6 females) participated in Experiment 3. Working memory was loaded with three colored squares that were presented for $500 \mathrm{~ms}$. The colors of the squares were randomly selected from a set of seven colors (red, green, blue, yellow, black, gray, and light blue). As in Experiments 1 and 2, the working memory trials were randomly placed within a particular hybrid search block.

\section{Experiments 4 and 5: Location memory}

Fourteen different observers participated in Experiments 4 and 5 (average age 29.2 years, 14 females). Following the methods of Oh and Kim (2004), in these experiments, the initial working memory displays consisted of three black squares $(500 \mathrm{~ms})$, and observers were told to remember the locations. In the test array, only a single square was presented. Observers were asked if this square was in a location that matched any of the three locations in the initial display. In Experiment 4, working memory trials were randomized among nonworking memory trials, whereas in Experiment 5, the working memory trials were blocked. The order of the WM load and no load blocks was counterbalanced between observers in Experiment 5.

\section{Experiment 6: Articulatory suppression}

Fourteen observers participated in Experiment 6 (average age 32.6, 6 females). Experiment 6 replicated Experiment 3 (colored square memory) with the following exceptions. Observers in this experiment were asked to memorize 2 or 16 objects in different blocks of the experiment. As in Experiment 5, WM load and no-load trials were blocked. The order of WM load and no load blocks was counterbalanced across observers. At the beginning of each block of visual search trials, observers were instructed to recite "abcd," "wxyz," "1234," or "6789" throughout that block of trials. The experimenter sat in the room with the observers to ensure that this phrase was audibly repeated throughout the experiment. The phrase for a given block was randomly permutated for each observer.

\section{Experiment 7: What is the cost of visual search on the VWM performance?}

The focus of Experiments 1-6 is the effect of WM load on hybrid search. Experiment 7 allowed us to look at the influence of hybrid search on WM capacity. Twenty-one observers participated in Experiment 7. One observer did not finish in the allotted time and was removed from further analyses (average age 26.6, 10 females). Experiment 7 replicated Experiment 3 (colored square memory) with the following exceptions. Observers in this experiment were asked to memorize 8 or 16 objects in different blocks of the experiment. There were three trial types in this experiment. Dual-task and No-Memory conditions were identical to trials in the previous experiments. Observers were also shown no-search trials. For these trials, the working memory load screen (three colored squares) preceded a screen that informed the observer that there was no search task on this trial and to press a button when ready to continue. All trial types were randomly interleaved with the block. 


\section{Results}

The results for Experiments 1-5 can be seen in Fig. 2 and Tables 1 and 2. During the course of seven experiments, we found very little evidence of an interaction between VWM load and target set size. For trials with a WM load, we focused our analyses on those trials where the WM task was answered correctly. We assessed three primary dependent measures of hybrid search performance: mean reaction time (RT), search efficiency (slope), and d' (sensitivity). Search efficiency was the measure of primary interest; RT and d' were essentially used as controls to ensure that if we did not find an effect of VWM load on slope of the RT $x$ set size functions, this was not due to a speed accuracy trade-off, or an overall accuracy decrement in the face of the VWM load. Previous evidence has suggested that RT increases as a linear function of the log of the memory set size (Wolfe, 2012; Cunningham \& Wolfe, 2014). Accordingly, we use a $\log _{2}$ scale for memory set size on the $\mathrm{X}$-axis on graphs throughout the paper.

To summarize, in each experiment we computed a $2 \times 4$ repeated measures ANOVA with WM load and target set size as factors. Not surprisingly, there was a large, reliable main effect of the number of targets held in ALTM (target set size) in each experiment. The VWM load had a less consistent effect. In Experiment 1 (the first object experiment) and Experiment 3 (the color experiment), there was a significant effect of WM load on reaction time, but only on absent trials (Exp1: 78-ms difference, $\mathrm{F}(1,12)=7.43, p=0.018$; Exp3: 275 -ms difference, $\mathrm{F}(1,11)=26.151, p<0.001)$. There was no main effect of WM load on RT in any of the other experiments. When present, the observed main effects on RT are broadly consistent with previous results (Oh \& Kim, 2004; Woodman \& Luck, 2004, 2007; Woodman, Vogel, \& Luck, 2001), although they are smaller and less reliable. With the exception of present trials in Experiment 3 (colored squares), search efficiency was unaffected by the presence or absence of a VWM load. This is in contrast to previous work that suggested that when VWM loads require observers to encode location, there is a decrease in search efficiency (Oh \& Kim, 2004; Woodman \& Luck, 2004). If the effect in Experiment 3 is reliable, perhaps it is the use of colored squares that is critical and was missing in the Experiments that failed to detect an interaction between search efficiency and VWM load. To test this hypothesis, observers in Experiments 6 and 7 were asked to memorize color squares. The efficiency X VWM load interaction did not replicate in either experiment. Thus, we found that there was a significant main effect of VWM load on slope for just 1 of the 6 (present or absent) possibilities using colored square memory and only 1 of the 14 total possibilities for all experiments. On balance, VWM load does not appear to reliably alter search efficiency in these experiments.
Finally, we found very little evidence for an interaction between memory set size in the hybrid search task and WM load. Of the five experiments for both present and absent trials, there was just one instance where the interaction was modestly statistically significant: present trials in Experiment $5: \mathrm{F}(3,33)=4.28, p=0.043$. We address this finding in more detail in the discussion of Experiments 6 and 7.

In each experiment, we computed both d' and c (criterion) to examine whether overall task performance or response bias varied systematically as a function of VWM load or target set size. there was a reliable effect of target set size (all Fs $>19$, all $p \mathrm{~s}<0.001$ ) but not VWM load (all Fs $<1.8$, all $p \mathrm{~s}>0.2$ ) on d' in Experiments 1-5. The $\mathrm{c}$ results are similar: there was a reliable effect of target set size in every experiment (all Fs $>2.9$, all $p$ s $<0.05$ ), and no effect VWM load (all Fs $<2.3$, all $p \mathrm{~s}>0.16)$ except in Experiment $1(\mathrm{~F}(1,12)=9.6$, $p<0.01)$. On the whole, these results suggest that while target set size reliably influenced both task difficulty and response bias (larger target set size led to a greater likelihood of "present" responses). VWM did not consistently influence either of these factors. This can be seen clearly in Fig. 3, which plots each observer's d' and criterion scores with and without a VWM load for Experiments 1-5.

\section{Bayes Factor}

Our primary interest was to determine whether holding information in working memory led to greater search difficulty when memory set size was increased. Whereas none of the interactions for this effect were significant for any of the three dependent variables (RT, slope, and d') in Experiments 1-5, it can be difficult to interpret null effects using traditional approaches (Wagenmakers, 2007). Accordingly, we used Bayes Factor (BF) calculations to evaluate whether the interaction between WM and memory set size was or was not contributing meaningfully to our results relative to a model that only included the main effects. We used the BayesFactor 0.9.6 package (Rouder \& Morey, 2012) in R, which implements the Jeffreys-Zellner-Siow (JZS) default on effect sizes (Rouder, Morey, Speckman, \& Province, 2012). This analysis yields a likelihood ratio that reflects the relative probability of the data arising from a pure main effect model compared to the interactive model. Thus, a BF of 4 can be interpreted as meaning that the model A is 4 times more likely to be supported than model B. Results are shown in Tables 1 and 2; large numbers indicate support for the main effect model. The preponderance of evidence favors the main effect model. In every case, the main effect model does a better job of explaining the data than the interactive model. This includes the present trials in Experiment 5, where the BF was 4.33 even though the interaction was modestly "significant" $(0.043)$ in the ANOVA. 



Blocked WM Task 
Fig. 2 Results from Experiments 1-5 denoting (from left to right) the materials used in the WM test, search efficiency (as measured by the slopes of the RT x visual set size for each memory set size), RT (averaged over visual set size), and d' as a function of memory set size. Note that the X-axis scale is logarithmic, rather than linear. See text for additional details. VWM performance (black line) is overlaid on the RT graph with proportion incorrect on the right axis. Critically, performance is very similar with (solid) and without (dashed) a working memory load. Error bars here and throughout the paper represent standard error of the mean

\section{Visual working memory performance}

The difficulty of the VWM task varied significantly across experiments (Fig. 2, second column). As expected, increasing the duration of the memory array from 0.5 to $3 \mathrm{~s}$ resulted in improved performance in Experiment $2(\mathrm{t}(24)=8.23, p<0.001)$. However, this difference in the amount of information that was successfully encoded into VWM had no influence on the interaction between the VWM load and the target set size. Similarly, although the color squares in Experiment 3 were easier to memorize than the locations in Experiment $4(\mathrm{t}(24)=5.32$, $p<0.001$ ), the additive model was preferred to the model with the interaction effect for all three of our dependent measures in both cases (Tables 1 and 2). Importantly, for our later discussion, there were no significant effects of memory set size on WM capacity (all $p>0.05$ ).

\section{Control Experiment 6: The role of verbal encoding}

Based on previous results, we were surprised to find that VWM load did not have a reliable effect on search slope in Experiments 1-5. Perhaps the absence of an effect was due to observers verbally encoding VWM information, thereby leaving the hybrid search task unaffected by this manipulation. If this were the case, we would expect that the addition of an articulatory suppression task (repeating a simple set of letters or numbers throughout the trials) would result in a large effect of VWM load on the search efficiency. One also might expect that this effect would interact with memory set size such that there would be a larger effect of VWM load when searching for a larger number of possible items. Experiment 6 examined this question using the colored square VWM stimuli. Results are shown, compared with the equivalent Experiment 3, in Fig. 4. Consistent with previous results, we found that although there was a large effect of memory set size on search efficiency (absent: $\mathrm{F}(1,13)=9.68$, $p<0.001$; present: $\mathrm{F}(1,13)=22.45, p<0.001)$, there was no effect of the presence of a VWM load (absent: $\mathrm{F}(1,13)=0.03$, $p=0.85$; present: $\mathrm{F}(1,13)=0.18, p=0.68)$ and the two factors did not interact reliably (absent: $\mathrm{F}(1,13)=0.6, p=0.44$; present: $\mathrm{F}(1,13)=0.78, p=0.39)$. These results suggest that the lack of influence of the VWM load on search efficiency in Experiments 1-5 was not driven by a verbal encoding strategy.

Table 1 Absent trials and d' results. Results of repeated measures ANOVAs and Bayes Factor estimation for Experiments 1-5

\begin{tabular}{|c|c|c|c|}
\hline & Slope & RT & d' \\
\hline \multirow[t]{3}{*}{ Object $(0.5 \mathrm{~s})$} & WM: F $(1,12)=3.94, p=0.071$ & WM: $\mathrm{F}(1,12)=7.43, p=0.018$ & $\mathrm{WM}: \mathrm{F}(1,12)=1.77, p=0.21$ \\
\hline & Mset: $\mathrm{F}(3,36)=6.64, p=0.001$ & Mset: $\mathrm{F}(3,36)=57.94, p<0.001$ & Mset: $\mathrm{F}(3,36)=19.33, p<0.001$ \\
\hline & $\begin{array}{l}\text { Interaction: } \mathrm{F}(3,36)=0.8411 \\
\quad p=0.480, \text { b.f. }=7.64\end{array}$ & $\begin{array}{c}\text { Interaction: } \mathrm{F}(3,36)=0.05 \\
\quad p=0.983 \text {, b.f. }=9.48\end{array}$ & $\begin{array}{c}\text { Interaction: } \mathrm{F}(3,36)=0.69 \\
p=0.56, \text { b.f. }=6.71\end{array}$ \\
\hline \multirow[t]{3}{*}{ Object (3 s) } & WM: F $(1,10)=0.114, p=0.742$ & WM: F $(1,10)=1.22, p=0.294$ & WM: F $(1,10)=0.008, p=0.92$ \\
\hline & Mset: $\mathrm{F}(3,30)=4.92, p=0.007$ & Mset: $\mathrm{F}(3,30)=53.19, p<0.001$ & Mset: $\mathrm{F}(3,30)=26.46, p<0.001$ \\
\hline & $\begin{array}{c}\text { Interaction: } \mathrm{F}(3,30)=0.61 \\
p=0.613, \text { b.f. }=4.88\end{array}$ & $\begin{array}{c}\text { Interaction: } \mathrm{F}(3,30)=1.12 \\
p=0.354, \text { b.f. }=7.77\end{array}$ & $\begin{array}{l}\text { Interaction: } \mathrm{F}(3,30)=0.419 \\
p=0.741, \text { b.f. }=7.10\end{array}$ \\
\hline \multirow[t]{3}{*}{ Color $(0.5 \mathrm{~s})$} & WM: F $(1,11)=0.227, p=0.642$ & WM: F $(1,11)=26.151, p<0.001$ & WM: $\mathrm{F}(1,11)=0.273, p=0.611$ \\
\hline & Mset: $\mathrm{F}(3,33)=22.878, p<0.001$ & Mset: $\mathrm{F}(3,33)=53.25, p<0.001$ & Mset: $\mathrm{F}(3,33)=43.86, p<0.001$ \\
\hline & $\begin{array}{c}\text { Interaction: } \mathrm{F}(3,33)=1.404 \\
p=0.258, \text { b.f. }=5.708\end{array}$ & $\begin{array}{c}\text { Interaction: } \mathrm{F}(3,33)=1.41 \\
p=0.258, \text { b.f. }=7.87\end{array}$ & $\begin{array}{l}\text { Interaction: } \mathrm{F}(3,33)=0.156 \\
\quad p=0.924, \text { b.f. }=7.891\end{array}$ \\
\hline \multirow[t]{3}{*}{ Location $(0.5 \mathrm{~s})$ interleaved } & WM: F $(1,13)=0.003, p=0.955$ & WM: F $(1,13)=1.628, p<0.224$ & WM: $\mathrm{F}(1,13)=0.004, p=0.951$ \\
\hline & Mset: $\mathrm{F}(3,39)=16.76, p<0.001$ & Mset: $\mathrm{F}(3,39)=51.50, p<0.001$ & Mset: $\mathrm{F}(3,39)=46.52, p<0.001$ \\
\hline & $\begin{array}{c}\text { Interaction: } \mathrm{F}(3,39)=2.54 \\
p=0.0706, \text { b.f. }=2.50\end{array}$ & $\begin{array}{c}\text { Interaction: } \mathrm{F}(3,39)=1.23 \\
p=0.311, \text { b.f. }=8.49\end{array}$ & $\begin{array}{l}\text { Interaction: } \mathrm{F}(3,39)=1.189 \\
\quad p=.326, \text { b.f. }=5.51\end{array}$ \\
\hline \multirow[t]{3}{*}{ Location $(0.5 \mathrm{~s})$ blocked } & WM: F $(1,11)=1.99, p=0.18$ & WM: F $(1,11)=0.0, p=0.998$ & WM: F $(1,11)=0.977, p=0.344$ \\
\hline & Mset: $\mathrm{F}(3,33)=8.05, p<0.001$ & Mset: $\mathrm{F}(3,33)=5.88, p<0.001$ & Mset: $\mathrm{F}(3,33)=25.08, p<0.001$ \\
\hline & $\begin{array}{l}\text { Interaction: } \mathrm{F}(3,33)=2.01 \\
\quad p=0.13, \mathrm{~b} . \mathrm{f} .=1.05\end{array}$ & $\begin{array}{c}\text { Interaction: } \mathrm{F}(3,33)=3.08, \\
p=0.082 \text {, b.f. }=8.12\end{array}$ & $\begin{array}{c}\text { Interaction: } \mathrm{F}(3,33)=0.85 \\
p=0.476, \text { b.f. }=6.77\end{array}$ \\
\hline
\end{tabular}


Table 2 Present trial results. Results of repeated measures ANOVAs and Bayes Factor estimation for Experiments 1-5

\begin{tabular}{|c|c|c|}
\hline & Slope & RT \\
\hline \multirow{3}{*}{ Object $(0.5 \mathrm{~s})$} & $\mathrm{WM}: \mathrm{F}(1,12)=0.410, p=0.534$ & $\mathrm{WM}: \mathrm{F}(1,12)=1.43, p=0.254$ \\
\hline & Mset: $\mathrm{F}(3,36)=44.7, p<0.001$ & Mset: $\mathrm{F}(3,36)=70.6, p<0.001$ \\
\hline & Interaction: $\mathrm{F}(3,36)=0.402, p=0.752$, b.f. $=5.24$ & Interaction: $\mathrm{F}(3,36)=0.104, p=0.957$, b.f. $=8.85$ \\
\hline \multirow[t]{3}{*}{ Object (3 s) } & $\mathrm{WM}: \mathrm{F}(1,10)=0.534, p=0.481$ & $\mathrm{WM}: \mathrm{F}(1,10)=0.03, p=0.865$ \\
\hline & Mset: $\mathrm{F}(3,30)=16.91, p<0.001$ & Mset: $\mathrm{F}(3,30)=56.72, p<0.001$ \\
\hline & Interaction: $\mathrm{F}(3,30)=1.55, p=0.220$, b.f. $=5.41$ & Interaction: $\mathrm{F}(3,30)=1.015, p=0.399$, b.f. $=5.79$ \\
\hline \multirow[t]{3}{*}{ Color (0.5 s) } & $\mathrm{WM}: \mathrm{F}(1,11)=9.766, p=0.0096$ & $\mathrm{WM}: \mathrm{F}(1,11)=19.6, p=0.001$ \\
\hline & Mset: $\mathrm{F}(3,33)=27.23, p<0.001$ & Mset: $\mathrm{F}(3,33)=55.88, p<0.001$ \\
\hline & Interaction: $\mathrm{F}(3,33)=0.774, p=0.516$, b.f. $=3.488$ & Interaction: $\mathrm{F}(3,33)=1.70, p=0.184$, b.f. $=6.23$ \\
\hline \multirow[t]{3}{*}{ Location $(0.5 \mathrm{~s})$ Interleaved } & $\mathrm{WM}: \mathrm{F}(1,13)=0.472, p=0.504$ & $\mathrm{WM}: \mathrm{F}(1,13)=1.23, p=0.287$ \\
\hline & Mset: $\mathrm{F}(3,39)=28.689, p<0.001$ & Mset: $\mathrm{F}(3,39)=60.85, p<0.001$ \\
\hline & Interaction: $\mathrm{F}(3,39)=2.104, p=0.115$, b.f. $=0.811$ & Interaction: $\mathrm{F}(3,39)=0.843, p=0.478$, b.f. $=7.49$ \\
\hline \multirow[t]{3}{*}{ Location (0.5 s) Blocked } & $\mathrm{WM}: \mathrm{F}(1,11)=0.07, p=0.796$ & $\mathrm{WM}: \mathrm{F}(1,11)=0.18, p=0.681$ \\
\hline & Mset: $\mathrm{F}(3,33)=36.54, p<0.001$ & Mset: $\mathrm{F}(3,33)=49.4, p<0.001$ \\
\hline & Interaction: $\mathrm{F}(3,33)=4.28, \boldsymbol{p}=\mathbf{0 . 0 4 3}$, b.f. $=4.33$ & Interaction: $\mathrm{F}(3,33)=3.14, p=0.314$, b.f. $=2.29$ \\
\hline
\end{tabular}

Note that the bolded p-value represents the only instance where slope significantly interacted with WM and Mset

Results from the RT and d' data from this Experiment 6 also were consistent with the general pattern in Experiments 1-5. There was a strong effect of memory set size for both measures (all $p<0.001$ ) but no main effect of VWM load (all $p>0.2$ ) or interaction between the two factors (all $p>0.1$ ).

\section{Experiment 7: Effects of hybrid search on working memory}

Experiments 1-6 make it quite clear that WM load does not have any consistent effect on hybrid search performance, but what about the other way around? Does performing a hybrid search during the retention period influence WM? It is notable

\section{D' across experiments}



that VWM in Experiment 1-5 is consistently lower than in previous published data involving three simple items (Luck \& Vogel, 1997). Moreover, it is interesting that WM performance is basically constant across memory set sizes in the hybrid search task. To determine whether this apparent decrement in VWM performance was caused by the presence of a hybrid search task during the retention interval, we performed an additional experiment that contained some trials that did not contain the hybrid search task.

In Experiment 7, one-third of trials did not contain a hybrid search Task, one-third did not have a VWM task, and onethird had both. When there was no hybrid search task, after encoding the VWM material, observers were instructed to

\section{Criterion across experiments}

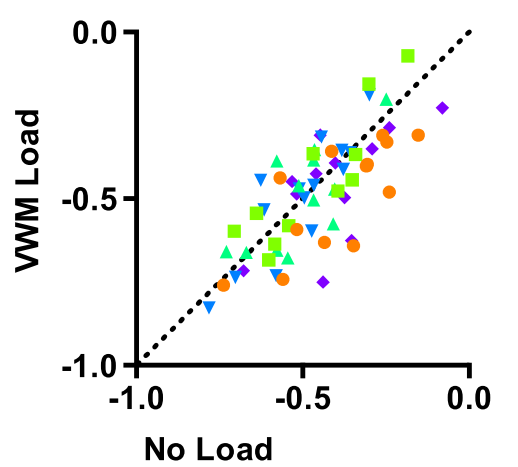

Fig. 3 D-prime and criterion data for Experiments 1-5. Across experiments, VWM load did not have a consistent effect on task difficulty or response bias 

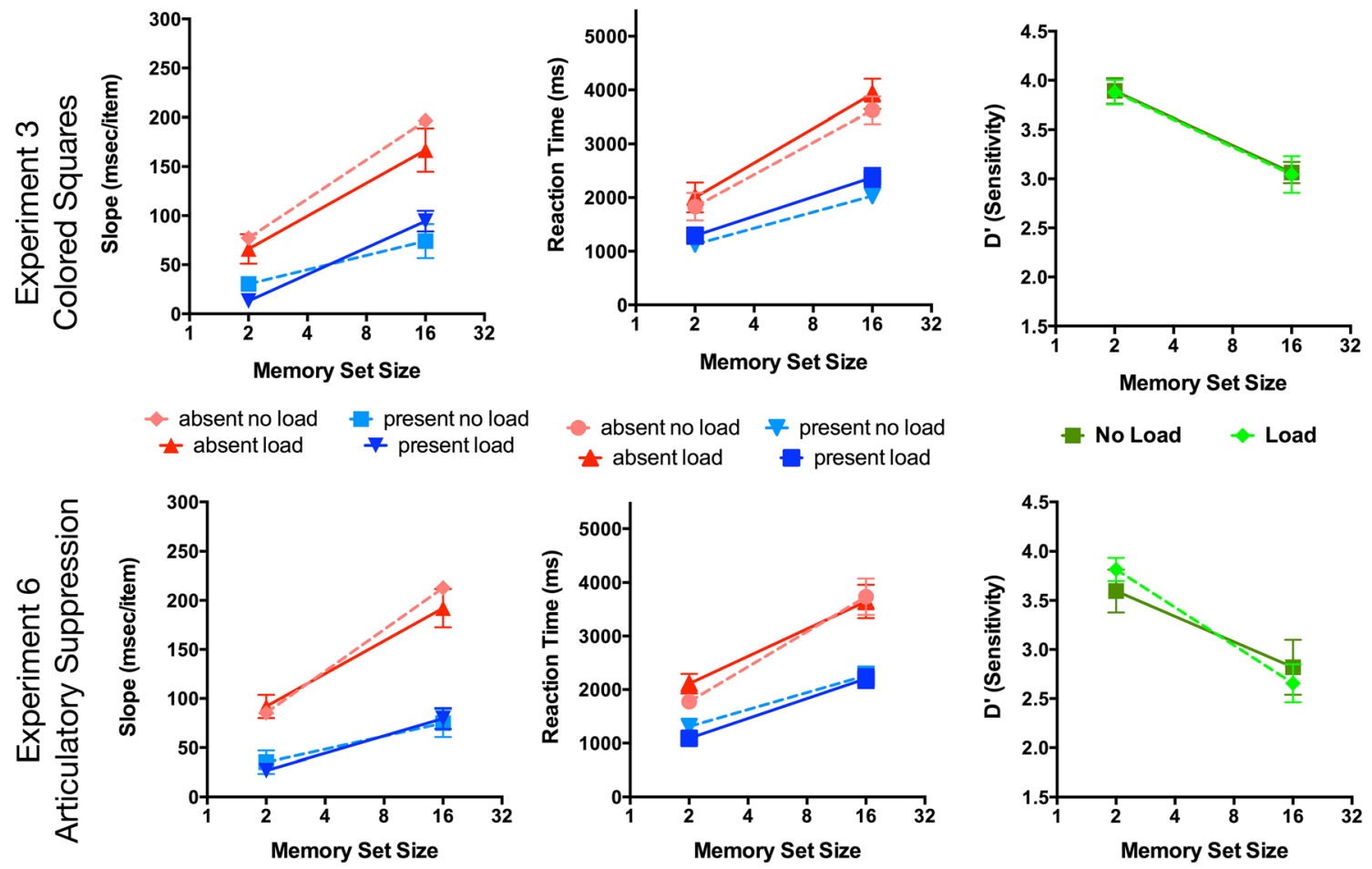

Fig. 4 Comparison between results for Experiments 3 and 6. The experiments were identical (including a VWM load of colored squares in both cases) aside from the articulatory suppression task observers

performed in Experiment 6. In addition, VWM trials were blocked, rather than interleaved, in Experiment 6

press a button to move on to the VWM test screen. All three trial types (Search Only, Memory Only, and Dual Task) were randomly interleaved. As in Experiments 3 and 6, the VWM load in this experiment was a set of colored squares.

The result from the Search Only and Dual Task trials are consistent with previous findings. There was a large effect of memory set size on search efficiency (absent: $\mathrm{F}(1,19)=4.61$, $p=0.04$; present: $\mathrm{F}(1,19)=9.52, p=0.006)$, no effect of the presence of a VWM load (absent: $\mathrm{F}(1,19)=0.77, p=0.39$; present: $\mathrm{F}(1,19)=1.95, p=0.18)$, and the two factors did not interact reliably (absent: $\mathrm{F}(1,19)=0.07, p=0.79$; present: $\mathrm{F}(1$, $19)=2.17, p=0.16$ ). However, VWM performance was strongly influenced by the presence of hybrid search task $(\mathrm{F}(1,19)=92.57, p<0.001)$. Performance in the absence of the search task was markedly higher (91\% correct, $k=2.46)$ than when the task was present ( $78 \%$ correct, $k=1.68$ ). Thus, while holding information in working memory does not appear to influence the efficiency with which we search through space during a hybrid search task, the act of performing a hybrid search task results in a the loss of about one "slot" worth of WM capacity (although this need not be a literal "slot." We make no commitments in that debate: Suchow, Fougnie, Brady, \& Alvarez, 2014). While it is clear that, consistent with previous results (Woodman \& Luck, 2010), there was dual-task interference, the interference appears to be a main effect on WM that does influence the efficiency with which the hybrid search task is performed.

\section{General discussion}

What do these results tell us about the role of WM in hybrid search and, perhaps, visual search more generally. Consider the outlines of a model, shown in Fig. 5.

In hybrid search, observers seek to determine if any of the items in the world are in the memory set of items, held in ALTM. Of course, the interaction of a visible item and its ALTM representation cannot be occurring in the world. The visible item must be selected by spatial attention. How and where is that representation compared to the target "templates" in ALTM? One possibility is that the current template is held in VWM and that the comparison is done in WM (Olivers et al., 2011). However, even if VWM can hold more than one template at a time (Gilchrist \& Cowan, 2011), one of the defining characteristics of WM is a severely limited capacity. It is therefore implausible that a memory set of 8,16 , or 64 items could be loaded into VWM in one step. Furthermore, if the comparison between the visual world and the memory set is occurring in VWM, one would expect that loading VWM with additional information should strongly influence the efficiency with which this process is performed. In the seven experiments outlined, we found no evidence of this. Thus, this most basic model can be ruled out.

Perhaps, VWM is the arena for the comparison of visual item and search template, but in the case of a large memory set, templates are swapped in and out of VWM from ALTM. 


\section{The World Spatial Attention VWM LTM (ALTM)}

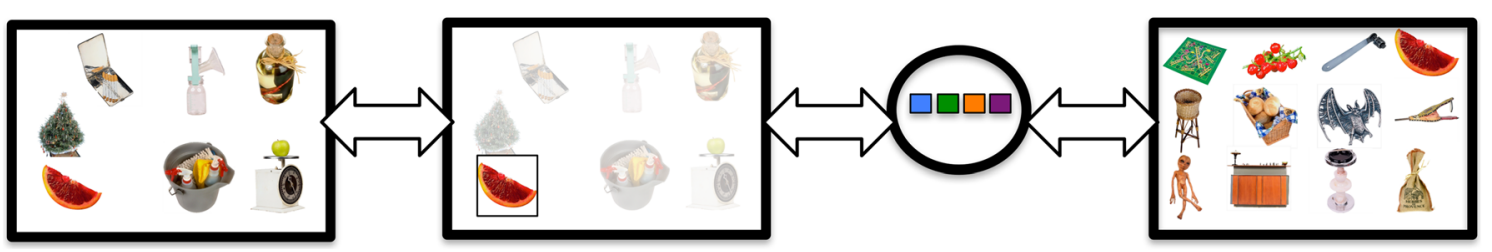

Fig. 5 In a hybrid search, observers determine if an item in the world matches any item in activate long-term memory (ALTM). We hypothesize that items are selected by visual attentional mechanisms

The problem with this account is that the number of required templates swaps would seem to be linear with the size of the memory set. Thus, it should take twice as many swaps to decide if the currently attended visual item is one of 16 items in memory than if it is one of 8. It is hard to see why such a linear process would produce RTs that were a logarithmic function of memory set size (Wolfe, 2012). Furthermore, the number of swaps necessary should be influenced by the presence or absence of VWM load, which would predict a strong effect of VWM load on search efficiency; an effect we do not see in any of the experiments reported here.

One could propose that the comparison is done in LTM. A visual representation is passed up the visual hierarchy to LTM, where it is matched against the ALTM representations of the memory set, bypassing VWM altogether. This, however, ignores the evidence that WM contents do influence visual search and, in particular for present purposes, the evidence of Experiment 7 that hybrid search produces a clear reduction in the WM capacity available to the change detection WM task.

An account that seems consistent with the current data proposes that WM is the narrow bottleneck that must be traversed by the visual representation of an item as it is moved from the visual system to LTM, where it can be compared to the ALTM representations of the memory set. VWM is, of course, very limited in capacity, so when a visual item must be moved through an already loaded VWM, it must displace the representation of some VWM information, effectively reducing the capacity of VWM. Thus, performance is reduced on the subsequent test of VWM. If we assume that a one-item channel must be maintained to continue moving visual item after visual item to LTM, we see that the cost of the hybrid search - VWM interaction will not be dependent on either the visual set size or the memory set size. It is simply the fixed cost of a one-item path through VWM. Thus, this account is consistent with the fixed, reduced VWM capacity seen in Experiments 1-5 (WM performance data in Fig. 2). It is consistent with the lack of an effect of VWM load on hybrid search, because in this situation hybrid search simply commandeers the same one-item path in all conditions. This account predicts that hybrid search (or any visual search) would be impossible, if one could force the observer not to and passed, one at a time, through visual working memory (VWM) to ALTM. In ALTM, the selected item is compared to the memory set in a process whose duration is a logarithmic function of the memory set size

relinquish any VWM capacity to search, although it is not currently clear how one would accomplish this goal.

In the present work, there is only exception to the general pattern that VWM load does not interfere with hybrid search efficiency. There was a modest effect on present (but not absent) trials in Experiment $5(p=0.043)$. The distinguishing manipulation in that experiment was that the VWM manipulation was blocked rather than interleaved. VWM trials were blocked in Experiment 7 as well, but there was no evidence of a statistically reliable interaction between VWM load and search efficiency in that experiment ( $p$ values for both present and absent trials $>0.3$ ). This suggests that the Experiment 5 finding may not be robust. However, there are prior studies where spatial working memory has been shown to interact reliably with visual search (Oh \& Kim, 2004; Woodman \& Luck, 2004). In the account offered, we propose that VWM was more resistant to surrendering the one-item pathway in those studies due to the inherent difference between hybrid search, where observers search for one potentially many possible targets amongst heterogeneous stimuli, and more traditional visual search, where observers learn to search for single target amongst relatively homogenous stimuli. This possibility needs further test.

Almost every model of visual search includes a role for WM (Bundesen, 1990; Desimone \& Duncan, 1995; Logan \& Gordon, 2001; Olivers et al., 2011; Wolfe, 1994). Typically, WM is thought to allow the observer to transfer small groups of items into WM for comparison with the target template. Contrary to this popular current view, the present results suggest that the target templates in hybrid search are not taking up space in VWM. As noted, this interpretation is implausible when there are large numbers of target templates as there must be in hybrid search with large memory set sizes. We have suggested that these templates are held in activated long-term memory (Cunningham \& Wolfe, 2014; Drew \& Wolfe, 2014; Wolfe, 2012). With the present results, we have clearly shown that search efficiency is not influenced by the presence or absence of a VWM load. We hypothesize that the role of WM in search is to serve as a conduit that passes visual representations to LTM where they can be compared to search template(s). In our experiments, that conduit remains open even when a VWM load is added. Apparently, the search task 
took priority such that the cost was paid by the WM task. We would predict that, if WM were disabled, hybrid search would fail.

While little is known about the role of WM in searching through ALTM, the link between LTM search and WM is relatively well understood. Retrieval from LTM is thought to involve both random and directed components (Shiffrin, 1970; Unsworth, Brewer, \& Spillers, 2013). Working memory seems to be important in selecting appropriate directed LTM search strategies (Raaijmakers \& Shiffrin, 1981; Unsworth et al., 2013). While high WM capacity observers are able to recall more items from an LTM category than low WM capacity observers during free recall, when search strategy was minimized via cued-recall, the differences disappeared (Unsworth et al., 2013).

It would be interesting to see if a difference between high and low WM capacity is seen in hybrid search. The current studies were not designed to directly address this question. It is possible that individuals with higher WM capacity (WMC) would be able to search more efficiently if some observers can pass items through VWM at a higher rate than others. Recent work by Anderson and colleagues supported this prediction (Anderson et al., 2013). The authors suggest that higher WMC observers can compare more items in parallel than lower WMC observers. In future work, it would be interesting to examine if WMC interacted with memory set size in these experiments.

In summary, in the experiments reported here, loading VWM had virtually no effect on hybrid search. In contrast, hybrid search had a clear, fixed effect on VWM, independent of memory set size in the hybrid task. We argue that VWM is, indeed, required for search tasks but that what is required is a path through VWM to pass a representation of the current object of attention to LTM, where that representation can be compared to templates stored in ALTM.

\section{References}

Anderson, D. E., Vogel E. K., \& Awh E. (2013). "A common discrete resource for visual working memory and visual search." Psychological Science, 24(6), 929-938.

Brady, T. F., Konkle, T., Alvarez, G. A., \& Oliva, A. (2008). Visual longterm memory has a massive storage capacity for object details. Proceedings of the National Academy of Sciences of the United States of America, 105(38), 14325-14329.

Bundesen, C. (1990). A theory of visual attention. Psychological Review, 97, 523-547.

Chelazzi, L., Duncan, J., Miller, E. K., \& Desimone, R. (1998). Responses of neurons in inferior temporal cortex during memoryguided visual search. Journal of Neurophysiology, 80, 2918-2940.

Cowan, N. (1995). Attention and memory: An integrated framework. Oxford University Press on Demand.
Cowan, N. (2001). The magical number 4 in short-term memory: A reconsideration of mental storage capacity. Behavioral and Brain Sciences, 24, 87-185.

Cunningham, C. A., \& Wolfe, J. M. (2014). The role of object categories in hybrid visual and memory search. Journal of Experimental Psychology: General, 143(4), 1585.

Desimone, R., \& Duncan, J. (1995). Neural mechanisms of selective visual attention. Annual Review of Neuroscience, 18, 193-222.

Downing, P., \& Dodds, C. (2004). Competition in visual working memory for control of search. Visual Cognition, 11(6), 689-703.

Drew, T., \& Wolfe, J. M. (2014). Hybrid search in temporal domain: Evidence for rapid, serial logarithmic search through memory. Attention, Perception, \& Psychophysics, 76, 296-303.

Forrin, B., \& Morin, R. E. (1969). Recognition times for items in shortand long-term memory. Acta Psychologica, 30, 126-141.

Gilchrist, A. L., \& Cowan, N. (2011). Can the focus of attention accommodate multiple, separate items? Journal of Experimental Psychology: Learning, Memory, and Cognition, 37(6), 1484-1502.

Lavie, N. (2005). Distracted and confused?: Selective attention under load. Trends in Cognitive Sciences, 9(2), 75-82.

Logan, G. D., \& Gordon, R. D. (2001). Executive control of visual attention in dual-task situations. Psychological Review, 108, 393-434.

Luck, S. J., \& Vogel, E. K. (1997). The capacity of visual working memory for features and conjunctions. Nature, 390, 279-281.

Luck, S. J., \& Vogel, E. K. (2013). Visual working memory capacity: From psychophysics and neurobiology to individual differences. Trends in Cognitive Sciences, 17(8), 391-400.

Miller, E. K., \& Cohen, J. D. (2001). An integrative theory of prefrontal cortex function. Annual Review of Neuroscience, 24, 167-202.

Oh, S. H., \& Kim, M.-S. (2004). The role of spatial working memory in visual search efficiency. Psychonomic Bulletin \& Review, 11(2), 275-281.

Olivers, C. N. L., Meijer, F., \& Theeuwes, J. (2006). Feature-based memory-driven attentional capture: Visual working memory content affects visual attention. Journal of Experimental Psychology-Human Perception and Performance, 32(5), 1243-1265.

Olivers, C. N., Peters, J., Houtkamp, R., \& Roelfsema, P. R. (2011). Different states in visual working memory: When it guides attention and when it does not. Trends in Cognitive Sciences, 15(7), 327-334.

Raaijmakers, J. G., \& Shiffrin, R. M. (1981). Search of associative memory. Psychological Review, 88(2), 93.

Rouder, J. N., \& Morey, R. D. (2012). Default Bayes factors for model selection in regression. Multivariate Behavioral Research, 47(6), 877-903.

Rouder, J. N., Morey, R. D., Speckman, P. L., \& Province, J. M. (2012). Default Bayes factors for ANOVA designs. Journal of Mathematical Psychology, 56(5), 356-374.

Schneider, W., \& Shiffrin, R. M. (1977). Controlled and automatic human information processing: I. Detection, search, and attention. Psychological Review, 84(1), 1-66.

Shiffrin, R. M. (1970). Memory search. In D. A. Norman (Ed.), Models of human memory (pp. 375-447). New York: Academic Press.

Soto, D., Hodsoll, J., Rotshtein, P., \& Humphreys, G. W. (2008). Automatic guidance of attention from working memory. Trends in Cognitive Sciences, 12(9), 342-348.

Suchow, J., Fougnie, D., Brady, T., \& Alvarez, G. (2014). Terms of the debate on the format and structure of visual memory. Attention, Perception, \& Psychophysics, 76(7), 2071-2079.

Unsworth, N., Brewer, G. A., \& Spillers, G. J. (2013). Working memory capacity and retrieval from long-term memory: The role of controlled search. Memory \& Cognition, 41(2), 242-254.

Wagenmakers, E. J. (2007). A practial solution to the pervasive problems of $p$ values. Psychonomic Bulletin \& Review, 14(5), 779-804.

Wolfe, J. M. (1994). Guided search 2.0: A revised model of visual search. Psychonomic Bulletin \& Review, 1, 202-238. 
Wolfe, J. M. (2012). Saved by a log: How do humans perform hybrid visual and memory search? Psychological Science, 23(7), 698-703.

Wolfe, J. M., Cave, K. R., \& Franzel, S. L. (1989). Guided search: An alternative to the feature integration model for visual search. Journal of Experimental Psychology: Human Perception and Performance, 15, 419-433.

Woodman, G. F., \& Luck, S. J. (2004). Visual search is slowed when visuospatial working memory is occupied. Psychonomic Bulletin \& Review, 11(2), 269-274.
Woodman, G. F., \& Luck, S. J. (2007). Do the contents of visual working memory automatically influence attetional selection during visual search? Journal of Experimental Psychology-Human Perception and Performance, 33(2), 363-377.

Woodman, G. F., \& Luck, S. J. (2010). Why is information displaced from visual working memory during visual search? Visual Cognition, 18(2), 275-295. doi:10.1080/13506280902734326

Woodman, G. F., Vogel, E. K., \& Luck, S. J. (2001). Visual search remains efficient when visual working memory is full. Psychological Science, 12(3), 219-224. 